\title{
Jinfukang induces cellular apoptosis through activation of Fas and DR4 in A549 cells
}

\author{
JUN LU ${ }^{1}$, JIAN CHEN $^{1}$, YANI KANG ${ }^{1,2}$, JUN WU $^{1}$, HUI SHI $^{3}$, YANLI FU ${ }^{4}$, LIJING JIAO ${ }^{2}$, \\ CHANGSHENG DONG ${ }^{2}$, XIAOWEI LI ${ }^{1}$, YU JIN ${ }^{3}$, WEI ZHAO ${ }^{5}$, LING XU ${ }^{2,6}$ and XIAODONG ZHAO ${ }^{1,2}$ \\ ${ }^{1}$ Shanghai Center for Systems Biomedicine, School of Biomedical Engineering and Bio-ID Center, \\ Shanghai Jiao Tong University, Shanghai 200240; ${ }^{2}$ Tumor Institute of Traditional Chinese Medicine, \\ Longhua Hospital, Shanghai University of Traditional Chinese Medicine, Shanghai 200032; \\ ${ }^{3}$ Engineering Research Center of Pharmaceutical Process Chemistry, Ministry of Education, School of Pharmacy, \\ East China University of Science and Technology, Shanghai 200237; ${ }^{4}$ Department of Oncology,
}

The Fourth Affiliated Hospital of Xinjiang Medical University, Xinjiang Medical University, Urumqi, Xinjiang 830000;

${ }^{5}$ Lab of Microbiology and Parasitology, Experimental Teaching Center, Shanghai Jiao Tong University School of Medicine,

Shanghai 200025; ${ }^{6}$ Department of Oncology, Yueyang Hospital of Integrated Traditional Chinese and Western Medicine,

Shanghai University of Traditional Chinese Medicine, Shanghai 200437, P.R. China

Received March 10, 2016; Accepted July 7, 2018

DOI: $10.3892 / \mathrm{ol} .2018 .9149$

\begin{abstract}
The traditional Chinese medicine Jinfukang (JFK) has been shown as a valuable drug to treat non-small cell lung cancer (NSCLC). Previously, it was reported that JFK-induced epigenetic alteration is involved in anti-lung cancer activity. In the present study, the effect of JFK on lung cancer cell lines was examined with the aim to further understand the underlying mechanisms of JFK-induced anti-lung cancer activity by transcriptome profiling analysis. JFK was observed to decrease lung cancer cell viability and simultaneously induce cellular morphology alteration. Additionally, this causes cell cycle arrest and apoptosis in A549 cells. The present RNA-seq analysis identified 5,281 genes with differential expression $(\mathrm{P}<0.05)$. Gene ontology analysis indicated that genes involved in the cell cycle pathway are downregulated, including cyclin-dependent kinase 2, cyclin-dependent kinase 4, cyclin B1 and cyclin A2, and apoptosis-associated genes are upregulated, including Fas, death receptor 4 (DR4), tumor protein P53 binding protein 2 and BCL2 interacting
\end{abstract}

Correspondence to: Dr Xiaodong Zhao, Shanghai Center for Systems Biomedicine, School of Biomedical Engineering and Bio-ID Center, Shanghai Jiao Tong University, 800 Dongchuan Road, Shanghai 200240, P.R. China

E-mail: xiaodongzhao@sjtu.edu.cn

Abbreviations: JFK, Jinfukang formula; NSCLC, non-small cell lung cancer; TCM, Traditional Chinese Medicine; RNA-Seq, RNA sequencing; CCK8, Cell Counting Kit 8; PI, propidium iodine; GO, gene ontology; KEGG, Kyoto Encyclopedia of Genes and Genomes

Key words: Jinfukang formula, traditional Chinese medicine, apoptosis, cell cycle arrest, non-small cell lung cancer, transcriptome protein 3 like. Particularly, the present results indicate knockdown of Fas and DR4 attenuates JFK-induced apoptosis in A549 cells. Overall, the present study suggests JFK induces cellular apoptosis through activation of Fas and DR4 in A549 cells and provides an insight for understanding the antitumor mechanisms of this Chinese traditional medicine.

\section{Introduction}

Lung cancer is one of the most malignant cancers, with the highest morbidity and mortality among all cancers in the world (1). Non-small cell lung cancer (NSCLC) is closely associated with a long history of tobacco smoking and accounts for $\sim 80 \%$ of all lung cancer cases (2). The primary treatment for patients with locally advanced or metastatic NSCLC includes systemic chemotherapy (alone or in combination with radiation therapy) and targeted biological therapy (3). However, lung cancer cells frequently show significant resistance to chemotherapeutic drugs (4). Therefore, it is urgent to develop alternative therapeutic strategies against lung cancer.

Traditional Chinese Medicine (TCM) has been effectively used against diseases for thousands of years in China $(5,6)$. Indeed, numerous Chinese medicinal herbs show promising effects in the treatment of cancers (7). Jinfukang (JFK), an Astragalus-based herbal formula consisting of 12 herbs (Table I), has been reported to have anti-tumor efficacy against NSCLC (8-10). It has been approved by the Chinese Food and Drug Administration as a drug against NSCLC. Although a previous study reported that JFK could inhibit proliferation and promote apoptosis of cancer cells in rats (9), the underlying mechanisms remain elusive.

As the majority of TCMs consist of various components, it is necessary to utilize high-throughput strategies to delineate the underlying mechanisms (11). Previous studies have shown that cDNA profiling may be effectively used to 
characterize the antitumor mechanism by measuring gene expression changes $(12,13)$. With the emergence of RNA-seq, this approach has provided a better platform to investigate the mechanisms of various drugs due to its performance in robustness and effectiveness $(14,15)$.

A previous study indicated that JFK-induced epigenetic alteration is involved in anti-lung cancer activity (16). In present study, the alteration in cellular growth and apoptosis upon JFK treatment was examined in human lung cancer cell lines, and RNA-seq analysis was performed to characterize the JFK-induced transcriptome. Among the genes whose expression was modulated by JFK, the present study further characterized those involved in the apoptosis pathway to understand how JFK exerts its effects on lung cancer cells.

\section{Materials and methods}

Preparation of JFK. JFK contains 12 herbals described in Table I, and these raw herbs were obtained from the pharmacy dispensary of Longhua Hospital, Shanghai University of Traditional Chinese Medicine (Shanghai, China). The names of herbs have been verified via www.theplantlist.org. Voucher specimens were deposited at the herbarium in Shanghai Jiao Tong University. The raw components of JFK (20 g) were minced and extracted with $200 \mathrm{ml} 70 \%$ ethanol at $80^{\circ} \mathrm{C}$ for $1 \mathrm{~h}$ according to the composition in Table I. Chromatographic and mass spectrographic finger prints for JFK ethanol extract were examined via LC-MS. The ethanol extract was filtered through a $0.45 \mu \mathrm{m}$ syringe filter and diluted to various concentrations used in the present study.

Cell culture. The human lung cancer A549, NCI-H1975, NCI-H1650 and NCI-H2228 cell lines were maintained with RPMI-1640 medium (Gibco; Thermo Fisher Scientific, Inc., Waltham, MA, USA) containing $10 \%$ fetal bovine serum (Gibco; Thermo Fisher Scientific, Inc.) and $100 \mathrm{U} / \mathrm{ml}$ penicillin and $100 \mu \mathrm{g} / \mathrm{ml}$ streptomycin (Thermo Fisher Scientific, Inc.) at $37^{\circ} \mathrm{C}$ in a humidified atmosphere containing $5 \% \mathrm{CO}_{2}$.

Cell viability analysis. Human lung cancer cell lines were treated with various concentrations of JFK extract $(0,0.041$, $0.054,0.081,0.108,0.162$ and $0.216 \mathrm{mg} / \mathrm{ml}$ ) for $48 \mathrm{~h}$ at $37^{\circ} \mathrm{C}$, and then the cell viability was evaluated using Cell Counting Kit 8 (CCK8; Dojindo Molecular Technologies, Inc., Kumamoto, Japan) by spectrophotometric plate reader (Omega Bio-Tek, Inc., Norcross, GA, USA). All cell viability results were tested by three independent experiments.

Morphological changes and DAPI staining. To evaluate the effects of JFK extract on cell growth, $1 \times 10^{5}$ A549 cells were seeded in each well of 6-well plates, and exposed to JFK extract at a half maximal inhibitory concentration (IC50; determined by CCK 8 assay) for $48 \mathrm{~h}$. The morphological changes in A549 cells were observed with a phase-contrast microscope (Model Ti-E; NIS4.0; Nikon, Tokyo, Japan). A549 cells were fixed, stained with DAPI and observed using fluorescence microscopy (Model Ti-E; NIS4.0; Nikon).

Cell cycle analysis. Briefly, A549 cells were harvested by trypsin with no EDTA (Thermo Fisher Scientific, Inc.), and washed twice with PBS. The cells were fixed with cold $70 \%$ ethanol overnight, and then stained with a solution consisting of $20 \mu \mathrm{g} / \mathrm{ml}$ propidium iodine (PI) (Sigma-Aldrich; Merck KGaA, Darmstadt, Germany) and 0.1\% TritonX-100 (Sigma-Aldrich; Merck KGaA), $100 \mu \mathrm{g} / \mathrm{ml}$ RNase A (Fermentas; Thermo Fisher Scientific, Inc.) for $15 \mathrm{~min}$ at $37^{\circ} \mathrm{C}$ in the dark. Cell cycle progression was then analyzed by flow cytometry (BD LSRFortessa; BD Biosciences, Franklin Lakes, NJ, USA) and ModFit LT software (version 3.2; Verity Software House, Topsham, ME, USA).

Cell apoptosis analysis. Determination of phosphatidyl serine (PS) and membrane integrity was performed using the Annexin V-FITC/PI Apoptosis kit (Beijing Zoman Biotechnology Co., Ltd., Beijing, China). In brief, A549, NCI-H1975, NCI-H1650 and NCI-H2228 cells were harvested by trypsin (no EDTA) and washed twice with PBS, then stained with Annexin V-FITC/PI and analyzed by flow cytometry as aforementioned. PI positive cells were designated as end stage apoptotic cells, and FITC-positive cells were designated as early stage apoptotic cells.

RNA-seq library construction. RNA-seq assays were performed in A549 cells without and with JFK treatment for $48 \mathrm{~h}(0.054 \mathrm{mg} / \mathrm{ml})$, as described in our recent study (17). Briefly, total RNA was extracted by TRIzol (Thermo Fisher Scientific, Inc.), and further treated with DNase to remove genomic DNA contamination. Isolation of mRNA was performed with Oligotex mRNA Mini kit (Qiagen, Hilden, Germany) and then used for RNA-seq library construction with NEBNext Ultra Directional RNA Library Prep kit for Illumina (New England BioLabs, Inc., Ipswich, MA, USA), which was subjected to Illumina sequencing. The raw sequencing data are available in the EMBL database (www.ebi.ac.uk/arrayexpress) under accession number E-MTAB-3592.

Analysis of differentially expressed genes. The raw sequencing reads were mapped to the reference genome (hg19) by TopHat. Cufflinks was used to characterize the differential transcription pattern (18). The present study has taken biases in library preparation into account. The gene expression level was measured by reads per kilobase of transcript per million reads mapped.

Functional annotation and pathway analysis. The Database for Annotation, Visualization and Integrated Discovery (DAVID) bioinformatics resource was used to annotate gene functions and pathways $(19,20)$.

Quantification of $m R N A$ level. Transcription level of genes of interest was evaluated using quantitative reverse transcription-quantitative polymerase chain reaction (RT-qPCR) with SYBR-Green PCR Master Mix (Qiagen). RT-qPCR was performed according to our previous studies $(13,16,17)$. All primer sequences used for RT-qPCR are presented in Table II.

Western blot analysis. Cells were washed with PBS and collected in a $1.5 \mathrm{ml}$ tube. A549 cells were lysed using the procedures essentially the same as described previously (13). The total protein extracts were obtained following 
Table I. Composition of JFK formula.

\begin{tabular}{|c|c|c|c|c|c|}
\hline Botanical name & Herbal name & Common name & Chinese name & Ratio & Voucher no. \\
\hline $\begin{array}{l}\text { Astragalu smembranaceus } \\
\text { (Fish.) Bunge }\end{array}$ & Radix astragali & Milkvetch root & Huang-qi & 8 & SJTU-JFK140121-1 \\
\hline $\begin{array}{l}\text { Glehnia littoralis } F \text {. } \\
\text { Schmidt ex Miq. }\end{array}$ & Radix glehniae & Coastal glehnia root & Bei-sha-shen & 8 & SJTU-JFK140121-2 \\
\hline $\begin{array}{l}\text { Asparagus cochinchinensis } \\
\text { (Lour.) Merr }\end{array}$ & Radix asparagi & $\begin{array}{l}\text { Cochinchinese } \\
\text { asparagus root }\end{array}$ & Tian-men-dong & 2 & SJTU-JFK140121-3 \\
\hline $\begin{array}{l}\text { Ligustrum lucidum } \\
\text { W.T. Aiton }\end{array}$ & $\begin{array}{l}\text { Fructus ligustri } \\
\text { lucidi }\end{array}$ & Glossy privet fruit & Nv-zhen-zi & 2 & SJTU-JFK140121-4 \\
\hline $\begin{array}{l}\text { Selaginella doederleinii } \\
\text { Hieron. }\end{array}$ & Herba selaginellae & Spikemoss & Shi-Shang-Bai & 8 & SJTU-JFK140121-5 \\
\hline $\begin{array}{l}\text { Paris polyphylla Smith var. } \\
\text { yunnanensis (Franch.) } \\
\text { Hand Mazz }\end{array}$ & $\begin{array}{l}\text { Rhizoma paridis } \\
\text { yunnanensis }\end{array}$ & $\begin{array}{l}\text { Yunnan manyleafparis } \\
\text { rhizome }\end{array}$ & Chong-lou & 3 & SJTU-JFK140121-6 \\
\hline $\begin{array}{l}\text { Epimedium sagittatum } \\
\text { (Siebold\&Zucc.) Maxim }\end{array}$ & Folium epimedii & Shorthornedepimedium & Yin-yang-huo & 2 & SJTU-JFK140121-7 \\
\hline $\begin{array}{l}\text { Gynostemma pentaphyllum } \\
\text { (Thunb.) Makino }\end{array}$ & $\begin{array}{l}\text { Herbal } \\
\text { gynostemmatis }\end{array}$ & $\begin{array}{l}\text { Five leaf gynostemma } \\
\text { herb }\end{array}$ & Jiao-gu-lan & 2 & SJTU-JFK140121-8 \\
\hline $\begin{array}{l}\text { Cornus officinalis Siebold } \\
\& \text { Zucc. }\end{array}$ & Fructus corni & $\begin{array}{l}\text { Asiatic cornelian } \\
\text { cherry fruit }\end{array}$ & Shan-zhu-yu & 2 & SJTU-JFK140121-9 \\
\hline Salvia chinensis Benth. & $\begin{array}{l}\text { Herba salviae } \\
\text { chinensis }\end{array}$ & Chinese sage & Shi-jian-chuan & 8 & SJTU-JFK140121-10 \\
\hline $\begin{array}{l}\text { Ophiopogon japonicas } \\
\text { (Thunb.) Ker Gawl }\end{array}$ & Radix ophiopogon & Dwarf lilyturf & Mai-dong tuber & 2 & SJTU-JFK140121-11 \\
\hline $\begin{array}{l}\text { Trigonella } \\
\text { foenum-graecum } \mathrm{L} \text {. }\end{array}$ & Semen trigonella & $\begin{array}{l}\text { Common fenugreek } \\
\text { seed }\end{array}$ & Hu-lu-ba & 2 & SJTU-JFK140121-12 \\
\hline
\end{tabular}

JFK, Jinfukang.

Table II. Primer sequences for reverse transcription-quantitative polymerase chain reaction.

Primer sequence

\begin{tabular}{|c|c|c|c|}
\hline Gene & GenBank & Forward $\left(5^{\prime}-3^{\prime}\right)$ & Reverse (5'-3') \\
\hline$C D K 2$ & [NG_034014] & TACACCCATGAGGTGACTCG & GTCCCCAGAGTCCGAAAGAT \\
\hline$C D K 4$ & [NM_000075] & CCCGAAGTTCTTCTGCAGTC & CTGGTCGGCTTCAGAGTTTC \\
\hline CCNA2 & [NM_001237] & GGTACTGAAGTCCGGGAACC & AAGATCCTTAAGGGGTGCAA \\
\hline$C C N B 1$ & [NM_172301] & TGTGGATGCAGAAGATGGAG & TTTGGTCTGACTGCTTGCTC \\
\hline DR4 & [NM_003844] & TGCAGGTCGTACCTAGCTCA & GGACACAACTCTCCCAAAGG \\
\hline Fas & [NG_009089] & ATTGCTCAACAACCATGCTG & CCAATCCCTTGGAGTTGATG \\
\hline TP53BP2 & [NG_029950] & TCCTTGGTCATTCAGGCTTC & GGCCAACTGGATGGATTTTA \\
\hline$B N I P 3 L$ & [NM_004331] & CAACAACAACTGCGAGGAAA & TTGCTGCTGTTCATGGGTAG \\
\hline$G A P D H$ & [NG_007073] & GCACCGTCAAGGCTGAGAAC & ATGGTGGTGAAGACGCCAGT \\
\hline
\end{tabular}

centrifugation $\left(18,000 \mathrm{x}\right.$ g for $15 \mathrm{~min}$, at $\left.4^{\circ} \mathrm{C}\right)$. The protein concentration was evaluated by Pierce BCA protein assay kit (Thermo Fisher Scientific, Inc.), according to the manufacturer's protocol. Protein samples $(\sim 50 \mu \mathrm{g})$ were fractionated by SDS-PAGE (10\% polyacrylamide gels) and transferred to a polyvinylidene fluoride membrane (EMD Millipore,
Billerica, MA, USA). The protein-binding membrane was incubated in PBS containing 0.1\% Tween-20 (Sigma-Aldrich; Merck KGaA, Darmstadt, Germany) for $60 \mathrm{~min}$ at $4^{\circ} \mathrm{C} .5 \%$ skim milk was used to block non-specific binding for $3 \mathrm{~h}$ at $4^{\circ} \mathrm{C}$. This was followed by incubation overnight at $4^{\circ} \mathrm{C}$ with primary antibody. The primary antibodies used in the present 
study included; cyclin B1 (CCNB1; cat no. D160234-0100; dilution, 1:250; Sangon Biotech Co., Ltd., Shanghai, China), cyclin-dependent kinase 4 (CDK4; cat no. 11026-1-AP; dilution, 1:100; Proteintech Group, Inc., Wuhan, China), death receptor 4 (DR4; cat no. 24063-1-AP; dilution, 1:100; Proteintech Group, Inc.), Fas (cat no. D220092-0100; dilution, 1:550; Sangon Biotech Co., Ltd.), and GAPDH (cat no. 5174S; dilution, 1:2,000; Cell Signaling Technology, Inc., Danvers, MA, USA). The membrane with protein antibody complexes were washed with PBS containing 0.1\% Tween-20, incubated for $2 \mathrm{~h}$ with a goat anti-rabbit horseradish peroxidaseconjugated secondary antibody (cat no. 14708S; dilution, 1:15,000; Cell Signaling Technology, Inc.) at $4^{\circ} \mathrm{C}$. Immunodetection was performed using ECL reagent (Santa Cruz Biotechnology Inc., Dallas, TX, USA) and images were captured and analyzed using the LI-COR Odyssey Infrared Imaging system (LI-COR Biosciences, Lincoln, NE, USA). The protein amounts were semi-quantified by analyzing blot intensity using GAPDH as loading control.

siRNA and transfection. siRNA duplexes were designed to downregulate DR4 and Fas. The DR4-targeting siRNA sequence was 5'-CCUUCAAGUUUGUCGUCGUdTdT-3' and the Fas-targeting siRNA sequence was 5'-GUGCAG AUGUAAACCAAACdTdT-3'. siRNAs for in vitro transfection were obtained from Shanghai Gene Pharma Co., Ltd. (Shanghai, China). Transfection was performed using Lipofectamine ${ }^{\circledR} 2000$ reagent (Invitrogen; Thermo Fisher Scientific, Inc., Waltham, MA, USA), according to the manufacturer's instructions. Briefly, A549 and NCI-H2228 cells were transfected with $50 \mathrm{nmol} / 1$ of siRNAs directed against Fas and DR4 respectively, and incubated for $6 \mathrm{~h}$ at $37^{\circ} \mathrm{C}$.

Statistical analysis. Data are presented as the mean \pm standard deviation. Comparisons between the groups were examined by standard one-way analysis of variance ANOVA, SPSS for Windows 14.0 software package (SPSS, Inc., Chicago, IL, USA), followed by Tukey's post hoc test. $\mathrm{P}<0.05$ was considered to indicate a statistically significant difference.

\section{Results}

JFK induces cell growth arrest and DNA damage in lung cancer cells. To examine the effects of JFK on lung cancer cell growth, A549, NCI-H1975, NCI-H1650 and NCI-H2228 cells were treated with JFK at various concentrations $(0,0.041,0.054$, $0.081,0.108,0.162$ and $0.216 \mathrm{mg} / \mathrm{ml}$ ) for $48 \mathrm{~h}$ and cell viability was assessed. The cell viability of these human lung cancer cell lines decreases significantly in a concentration-dependent manner following JFK treatment (Fig. 1A). Additionally, JFK was observed to exert an increased inhibition effect on A549 cells compared with other three human lung cancer cell lines, with a IC50 of $\sim 0.054 \mathrm{mg} / \mathrm{ml}$. JFK treatment $(0.054 \mathrm{mg} / \mathrm{ml})$ for $48 \mathrm{~h}$ reshapes a large number of A549 cells to be distinctly flat and enlarged in size (Fig. 1B) and makes the nucleus condensed and disintegrated (Fig. 1C), indicating multiple effects of JFK on A549 cells. Based on these observations, A549 was next focused on and the antitumor mechanisms of JFK were characterized.
JFK leads to cell cycle arrest and cell apoptosis in A549 cells. To further investigate whether JFK cytotoxicity involves cell cycle arrest, a flow cytometry assay was performed to characterize alterations of the cell cycle. As shown in Fig. 1D, cell cycle analysis indicates that a significant portion of A549 cells accumulates in G1 and G2 phase upon JFK $(0.054 \mathrm{mg} / \mathrm{ml})$ treatment, suggesting that JFK could induce G1/S and G2/M transition arrest. To examine whether JFK triggers apoptosis in A549 cells, the extent of apoptotic cells was investigated by flow cytometry, and it was observed that numerous A549 cells were induced to undergo apoptosis after exposure to JFK for $48 \mathrm{~h}$ (Fig. 1E). This phenomenon not only presents in early apoptosis, but also presents in total apoptosis (Fig. 1F). Thus, the cell apoptotic rates dramatically increase upon JFK treatment.

JFK-associated transcriptome analysis in A549 cells. Given the observations of anti-proliferation and pro-apoptosis effects induced by JFK, the present study subsequently aimed to understand the underlying molecular mechanisms by transcriptome-wide differential gene expression analysis through RNA-seq. In total, 13,130,096 and 10,433,359 reads were generated from JFK-treated and untreated A549 cells, 54 and $66 \%$ of which were uniquely mapped to the human genome, corresponding to 13,720 and 17,519 expressed genes, respectively (Table III). Compared with the control, 5,281 differentially expressed genes $(\mathrm{P}<0.05)$ were identified, with $2,777(52.6 \%)$ downregulated and 2,504 (47.4\%) upregulated (Fig. 2A).

Gene ontology analysis of differentially expressed genes. Gene Ontology (GO) analysis of the genes with JFK-induced differential expression was performed using DAVID (19) and Kyoto Encyclopedia of Genes and Genomes (KEGG) pathways database (20). The top ten GO items were listed in Table IV, in which apoptosis-associated and cell cycle-associated biological processes are statistically enriched in the upregulated and downregulated genes, respectively. For the gene sets with differential expression, KEGG pathway analysis was performed. Similarly, it was observed that the cell cycle and apoptosis pathways are also included in the top 10 enriched pathways (Fig. 2B and C). These results suggest that JFK could induce growth inhibition potentially through repressing pathways of cell cycle, nuclear division and translation pathways. Simultaneously, it could induce apoptosis in A549 cells partially through activating apoptosis, cell death and protein transport pathways.

JFK modulates the expression of genes involved in cell cycle and apoptosis. For gene sets with a differential expression pattern determined by RNA-seq analysis, cell cycle-associated genes, including $C D K 2, C D K 4, C C N B 1$ and cyclin A2 (CCNA2), and apoptosis-associated genes, including Fas, $D R 4$, tumor protein $\mathrm{P} 53$ binding protein 2 (TP53BP2) and BCL2 interacting protein 3 like $(B N I P 3 L)$, for further validation (Fig. 3A). The transcriptional activities of selected genes were measured by RT-qPCR, and the results were shown in Fig. 3B and C. These results confirm that JFK represses cell cycle-associated genes, and activates apoptosis-associated genes as well. Among these selected genes, the alterations in 
A

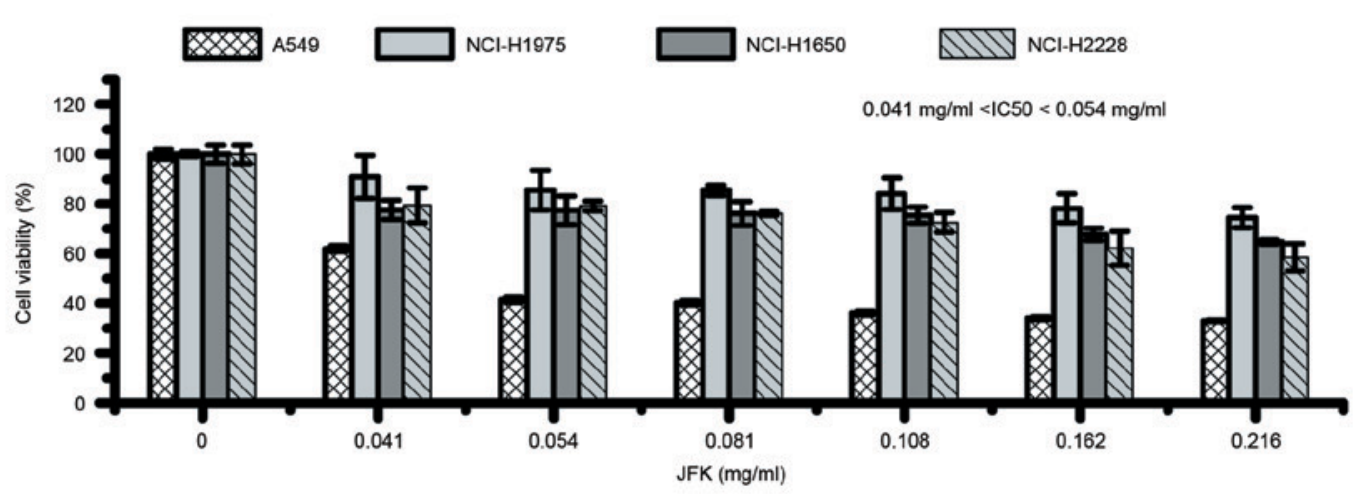

B
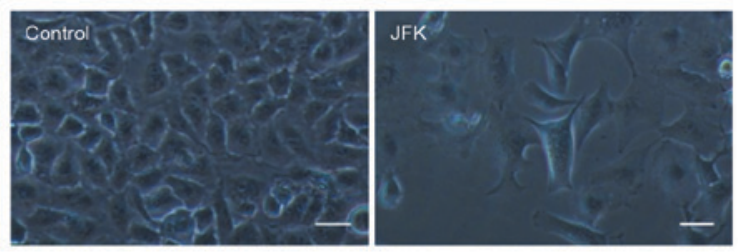

D
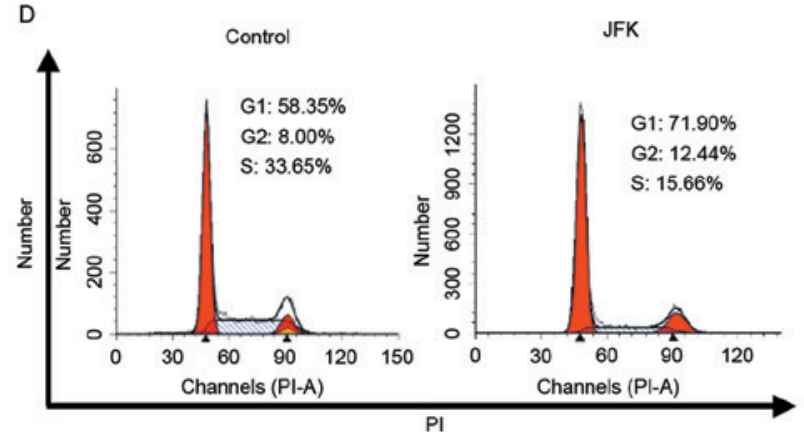

F

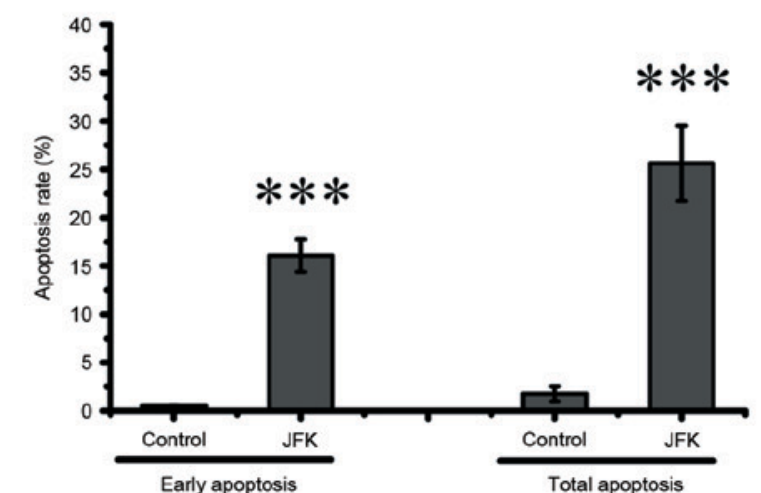

C
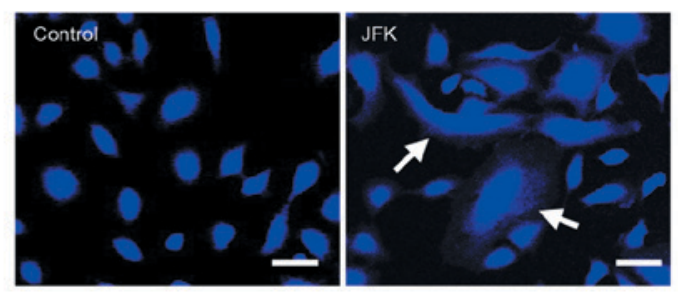

E

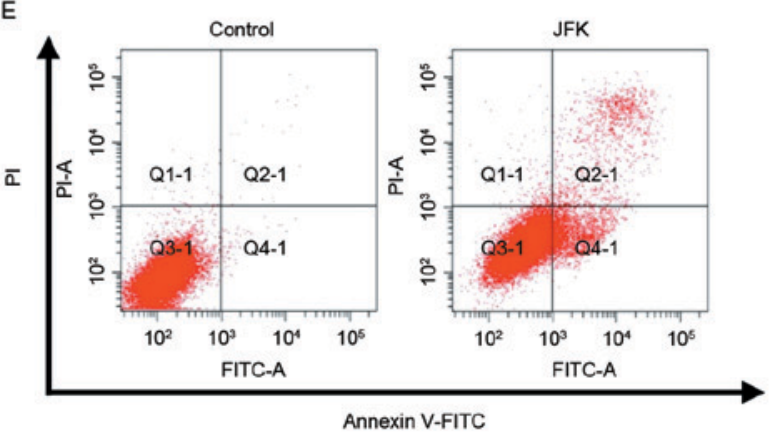

Figure 1. Effect of JFK on cytotoxicity in lung cancer cells. (A) A549, NCI-H1975, NCI-H1650 and NCI-H2228 cells were treated with JFK (0, 0.041, 0.054, $0.081,0.108,0.162$ and $0.216 \mathrm{mg} / \mathrm{ml}$, respectively) for $48 \mathrm{~h}$, and examined for viability. Data are expressed as the mean \pm SD $(\mathrm{n}=3)$. (B) A549 cells were exposed to JFK $(0.054 \mathrm{mg} / \mathrm{ml})$ for $48 \mathrm{~h}$. Morphological alteration was examined by phase-contrast microscopy. Scale bar, $50 \mu \mathrm{m}$. (C) A549 cells were stained with DAPI for detecting nucleus changes using fluorescence microscopy. Scale bar, $25 \mu \mathrm{m}$. (D) Cell cycle distribution was evaluated by flow cytometry. (E) Apoptotic cells were determined by flow cytometry after exposure to JFK for $48 \mathrm{~h}$. (F) Ratios of early apoptosis and total apoptosis were analyzed basing on flow cytometric detection. Data are expressed as the mean $\pm \mathrm{SD}(\mathrm{n}=3) .{ }^{* * *} \mathrm{P}<0.001$. SD, standard deviation; JFK, Jinfukang; PI, propidium iodide; FITC, fluorescein isothiocyanate; IC50, half maximal inhibitory concentration.

protein level of $C D K 4, C C N B 1$, Fas and $D R 4$ were examined by western blot analysis. As shown in Fig. 3D and E, protein levels of $C D K 4$ and $C C N B 1$ decrease and those of Fas and $D R 4$ increase after JFK $(0.054 \mathrm{mg} / \mathrm{ml})$ treatment. 
Table III. The statistics of RNA-seq data sets.

\begin{tabular}{lcccc}
\hline Group & Total reads & Unique mapping reads & Mapping rate, $\%$ & Expressed gene number \\
\hline Control & 10433359 & 6908569 & 66 & 17519 \\
JFK & 13130096 & 7035144 & 54 & 13720 \\
\hline
\end{tabular}

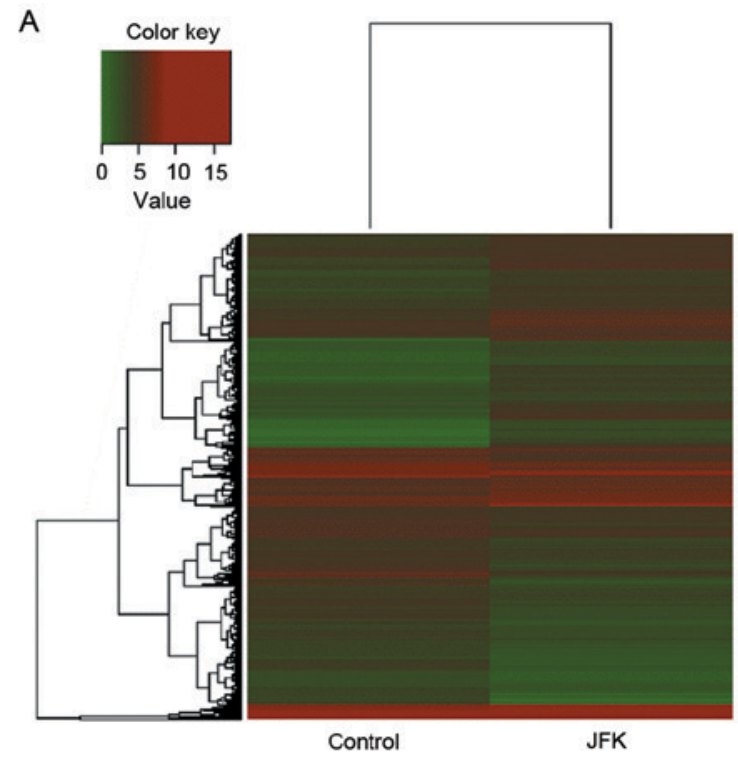

B

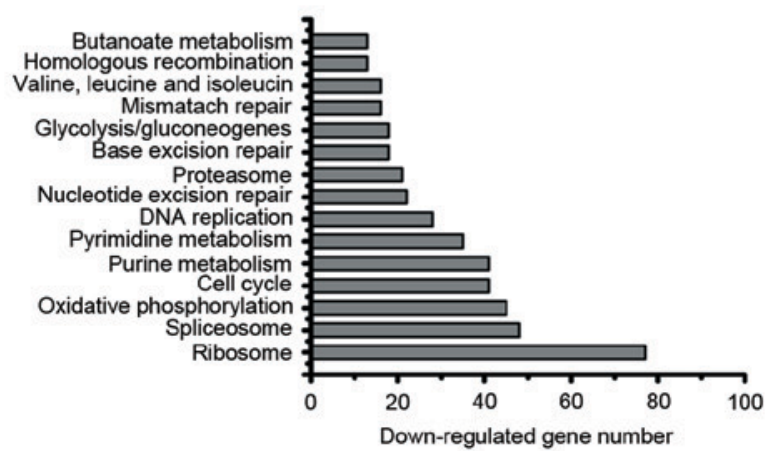

C

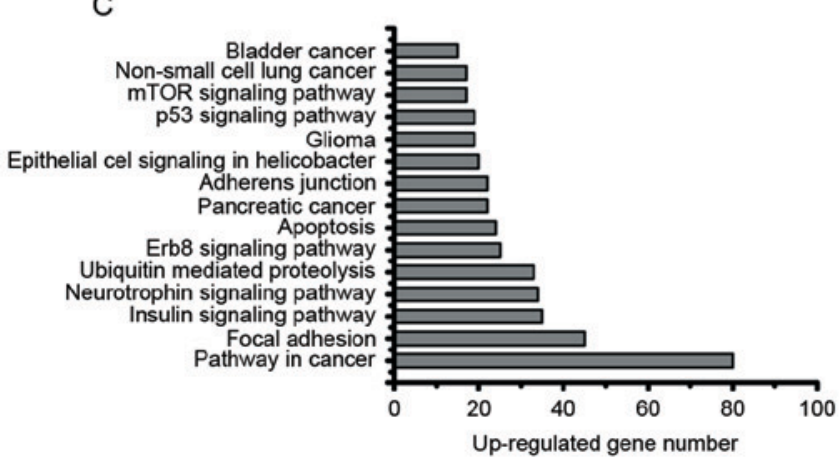

Figure 2. Functional classification and pathway analysis of differentially expressed genes. (A) Heat map of RNA-seq analysis in A549 cells with or without JFK $(0.054 \mathrm{mg} / \mathrm{ml})$ treatment. In total, 5,281 genes were identified as differentially expressed $(\mathrm{P}<0.05)$. (B and $\mathrm{C}) \mathrm{KEGG}$ pathway analysis of (B) upregulated and (C) downregulated genes. JFK, Jinfukang.
Table IV. GO analysis of differentially expressed genes.

A, Downregulated

\begin{tabular}{llrl}
\hline Gene ID & \multicolumn{1}{c}{ Function } & Count & P-value \\
\hline GO:0006414 & Translational elongation & 85 & $6.79 \times 10^{-57}$ \\
GO:0006412 & Translation & 140 & $6.93 \times 10^{-39}$ \\
GO:0007049 & Cell cycle & 226 & $2.69 \times 10^{-32}$ \\
GO:0022402 & Cell cycle process & 178 & $7.65 \times 10^{-30}$ \\
GO:0000278 & Mitotic cell cycle & 132 & $7.16 \times 10^{-28}$ \\
GO:0000279 & M phase & 120 & $2.39 \times 10^{-26}$ \\
GO:0022403 & Cell cycle phase & 134 & $1.00 \times 10^{-23}$ \\
GO:0000087 & M phase of mitotic cell & 88 & $5.95 \times 10^{-22}$ \\
& cycle & & \\
GO:0000280 & Nuclear division & 87 & $6.22 \times 10^{-22}$ \\
GO:0007067 & Mitosis & 87 & $6.22 \times 10^{-22}$ \\
\hline
\end{tabular}

B, Upregulated

\begin{tabular}{llrl}
\hline Gene ID & \multicolumn{1}{c}{ Function } & Count & P-value \\
\hline GO:0046907 & Intracellular transport & 150 & $5.33 \times 10^{-15}$ \\
GO:0015031 & Protein transport & 166 & $1.36 \times 10^{-14}$ \\
GO:0045184 & Establishment of protein & 167 & $1.51 \times 10^{-14}$ \\
& localization & & \\
GO:0008104 & Protein localization & 182 & $1.28 \times 10^{-13}$ \\
GO:0010941 & Regulation of cell death & 165 & $1.09 \times 10^{-11}$ \\
GO:0042981 & Regulation of apoptosis & 163 & $1.32 \times 10^{-11}$ \\
GO:0043067 & Regulation of programmed & 164 & $1.55 \times 10^{-11}$ \\
& cell death & 149 & $3.69 \times 10^{-11}$ \\
GO:0016265 & Death & 148 & $4.29 \times 10^{-11}$ \\
GO:0008219 & Cell death & 95 & $4.45 \times 10^{-10}$ \\
GO:0034613 Cellular protein localization & & \\
\hline
\end{tabular}

GO, gene ontology.

Activation of Fas and DR4 contributes to JFK-induced pro-apoptotic effect in A549 cells. To explore whether Fas and DR4 mediate the JFK-induced apoptosis in A549 cells, lastly siRNA assays were performed to suppress the expression of Fas and DR4 in JFK-treated A549 cells. As shown in Fig. 4A and $\mathrm{B}$, the apoptotic rates (including early apoptosis rate and total apoptosis rate) significantly decrease when Fas and DR4 were knocked down. The protein expression levels of Fas and DR4 also drop when Fas and DR4 were knocked down (Fig. 4C). These results indicate that activation of Fas and DR4 is partially involved in JFK-induced apoptosis. 
A

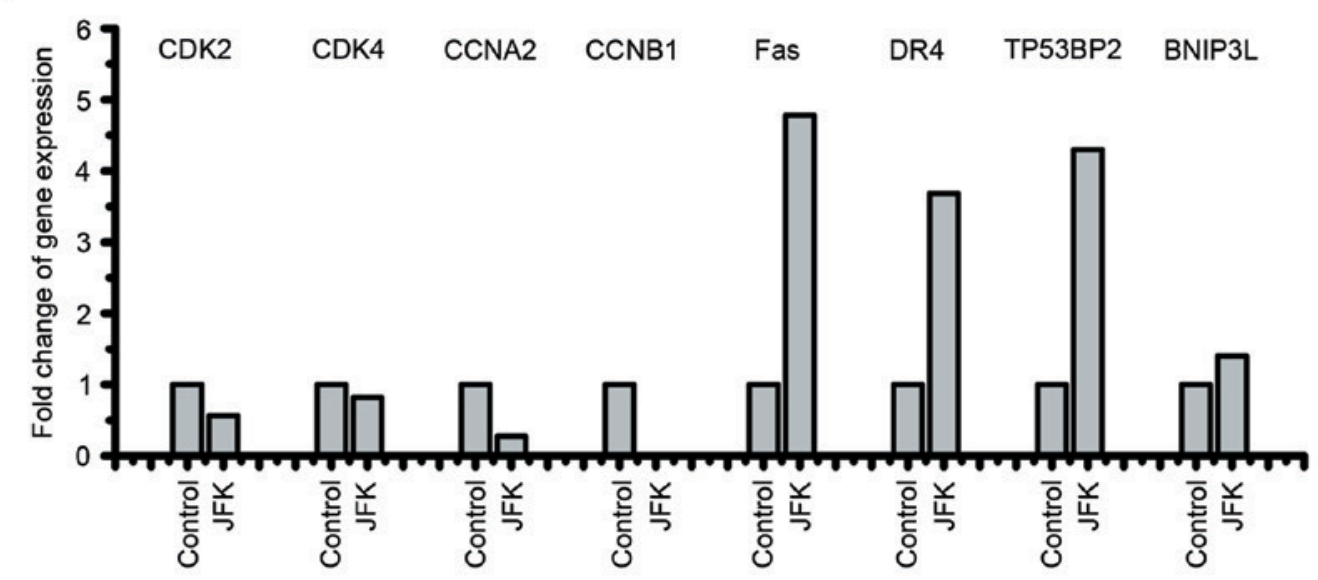

B

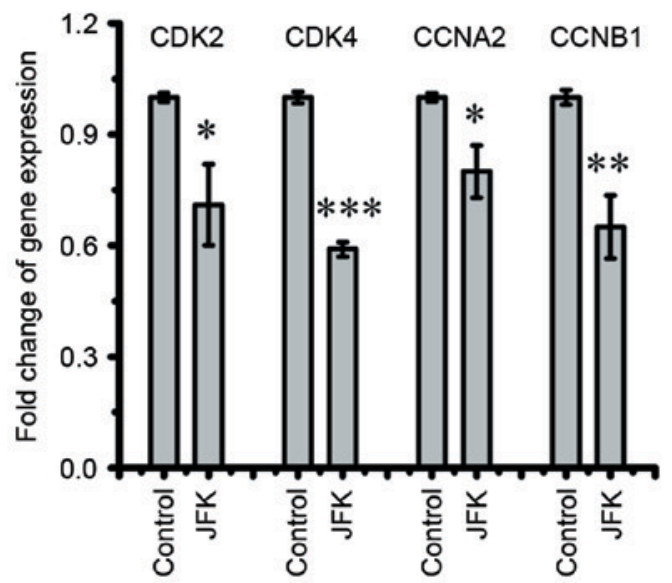

D

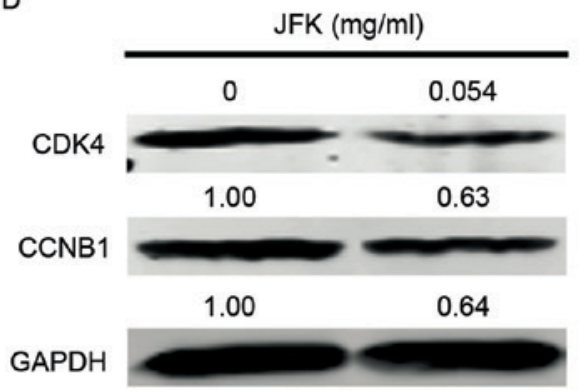

C

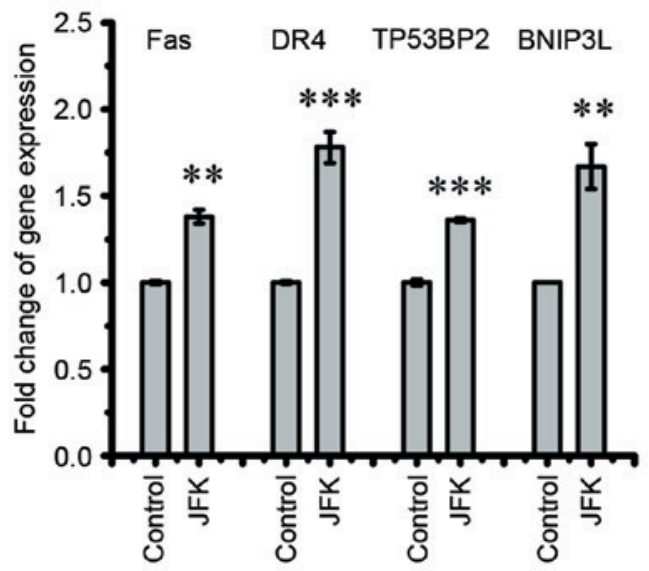

E

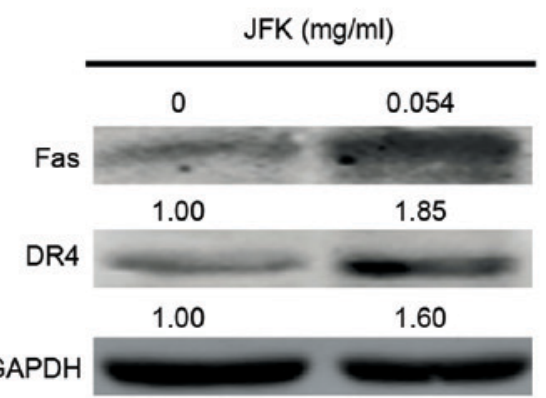

Figure 3. Expression validation of genes involved in the cell cycle and apoptosis upon JKF treatment in A549 cells. (A) Fold-change of cell cycle and cell apoptosis-associated gene expression from RNA-seq results. (B and C) A549 cells were treated with JFK (0 or $0.054 \mathrm{mg} / \mathrm{ml})$ for $48 \mathrm{~h}$, and then the mRNA levels of cell cycle and apoptosis-associated genes were examined by reverse transcription-quantitative polymerase chain reaction. The data are expressed as the mean \pm standard deviation $(\mathrm{n}=5)$. ${ }^{*} \mathrm{P}<0.05,{ }^{* *} \mathrm{P}<0.01$ and ${ }^{* * *} \mathrm{P}<0.001$. (D and E) A549 cells were treated with or without JFK $(0.054 \mathrm{mg} / \mathrm{ml})$ for $48 \mathrm{~h}$, and then the protein levels of $C D K 4, C C N B 1$, Fas and DR4 were examined by western blot analysis. CDK4, cyclin-dependent kinase 4; CDK2, cyclin-dependent kinase 2; CCNB1, cyclin B1; CCNA2, cyclin A2; TP53BP2, tumor protein P53 binding protein 2; BNIP3L, BCL2 interacting protein 3 like; JFK, Jinfukang.

\section{Discussion}

Herbal intervention has been widely used for the treatment and prevention of various diseases for thousands of years in China (21). The majority of known anticancer drugs are derived from herbal plants (22). JFK, a type of Astragalus-based herbal formula, has been reported to be clinically effective in
NSCLC patients $(8,10,23)$. In the present study, the anticancer mechanisms of JFK were explored. The cellular behavior of lung cancer cell lines treated with JFK were examined, and it was found that JFK could induce cellular growth arrest and the cell cycle is regulated by a complex cascade of events and mediated by cell cycle regulatory proteins, including cyclins and CDKs (24). CDK2 and CDK4 are two 
A
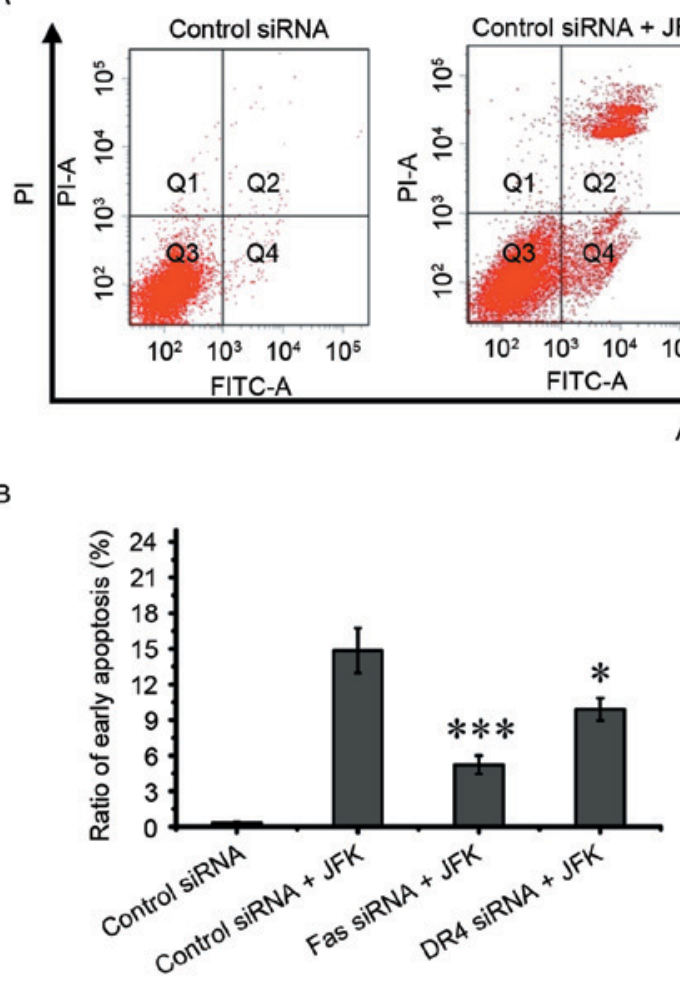

C
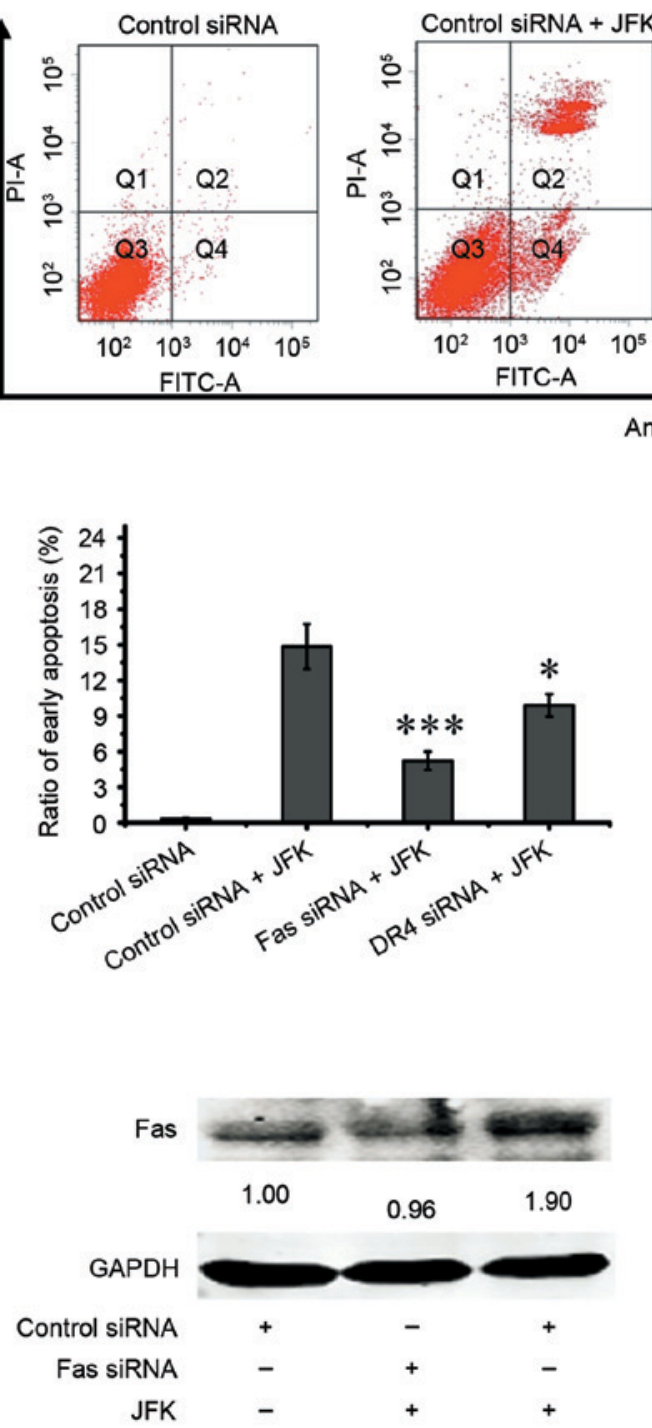
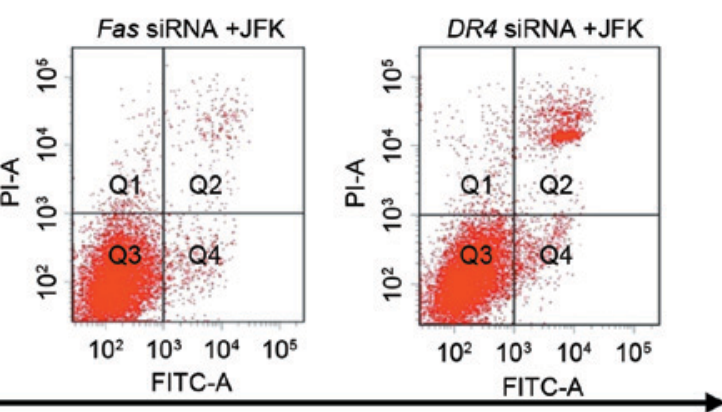

Annexin V-FITC
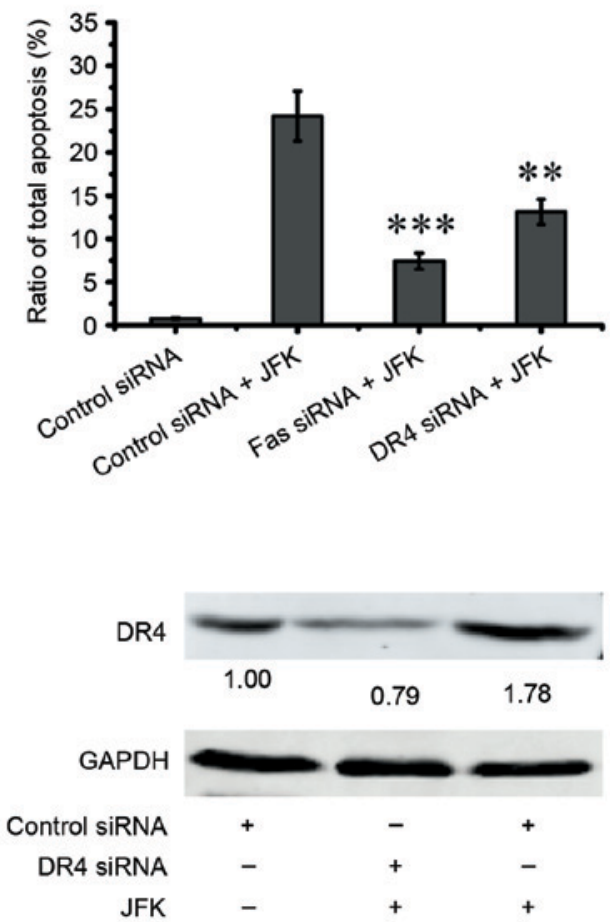

Figure 4. Knockdown of DR4 and Fas attenuates the pro-apoptotic effect of A549 cells induced by JFK. Fas and DR4 in A549 cells were knocked down, and the cells were exposed to JFK $(0.054 \mathrm{mg} / \mathrm{ml})$ for $48 \mathrm{~h}$. (A) The cell apoptosis ratio was examined by flow cytometry. (B) Ratios of early apoptosis and total apoptosis were analyzed basing on flow cytometry detection. Data are expressed as the mean \pm standard deviation $(\mathrm{n}=3),{ }^{*} \mathrm{P}<0.05,{ }^{* *} \mathrm{P}<0.01$ and ${ }^{* * *} \mathrm{P}<0.001$. (C) Fas and DR4 protein expression levels were determined by western blot analysis. DR4, death receptor 4; siRNA, small interfering RNA; JFK, Jinfukang; PI, propidium iodide; FITC, fluorescein isothiocyanate.

key members of the cyclin-dependent kinase family, whose activities are restricted to the G1-S phase, and are essential for the G1-Stransition $(25,26)$. CCNA2 is a critical regulatory factor that can interact with CDK kinases for regulating G1-S transition and G2-M transition (27). CCNB1 usually has an important role in mitosis, and indicates a poor prognosis when overexpressed in NSCLC (28). The present results show that A549 cells could be induced to a state of G1 and G2 phase enrichment upon JFK treatment (Fig. 1D), accompanying downregulation of cell cycle-associated genes, including $C D K 2, C D K 4, C C N B 1$ and $C C N A 2$ (Figs. 3A and 4B). These results suggest that JFK leads to A549 cell cycle arrest through the suppression of the cell cycle pathway.

Induction of apoptosis in tumor cells is one of the most common anticancer mechanisms in cancer therapies (29). The death receptor apoptotic pathway has previously been proposed as an anti-cancer drug target in human lung cancer (30). Fas and $D R 4$, two members of the death receptor family, have a vital role during the process of apoptosis. TP53BP2 induces apoptosis through the mitochondrial death pathway (31). BNIP3L has been reported to promote cell death via apoptosis (32). In the present study, it was found the expression activities of Fas and DR4 in A549 cells are upregulated in both mRNA and protein levels after JFK treatment. TP53BP2 and BNIP3L are also activated by JFK (Fig. 3A and C). Particularly, it was observed that downregulation of Fas and DR4 could attenuate JFK-induced apoptosis in A549 cells (Fig. 4). However, this phenomenon was not observed to occur in the human lung cancer NCI-H1650 and NCI-H228 cell lines, although JFK also exerts an inhibitory viability effect on these two cell lines (Fig. 5A-C). JFK enhances the transcription of DR4 and Fas in NCI-H2228 cells, but induces marginal apoptosis in siRNA knockdown assays (Fig. 5D-F), suggesting JFK-induced apoptosis is cellular context-dependent.

Overall, the present study reported that JFK induces cellular growth arrest and apoptosis in lung cancer cells. The 
A

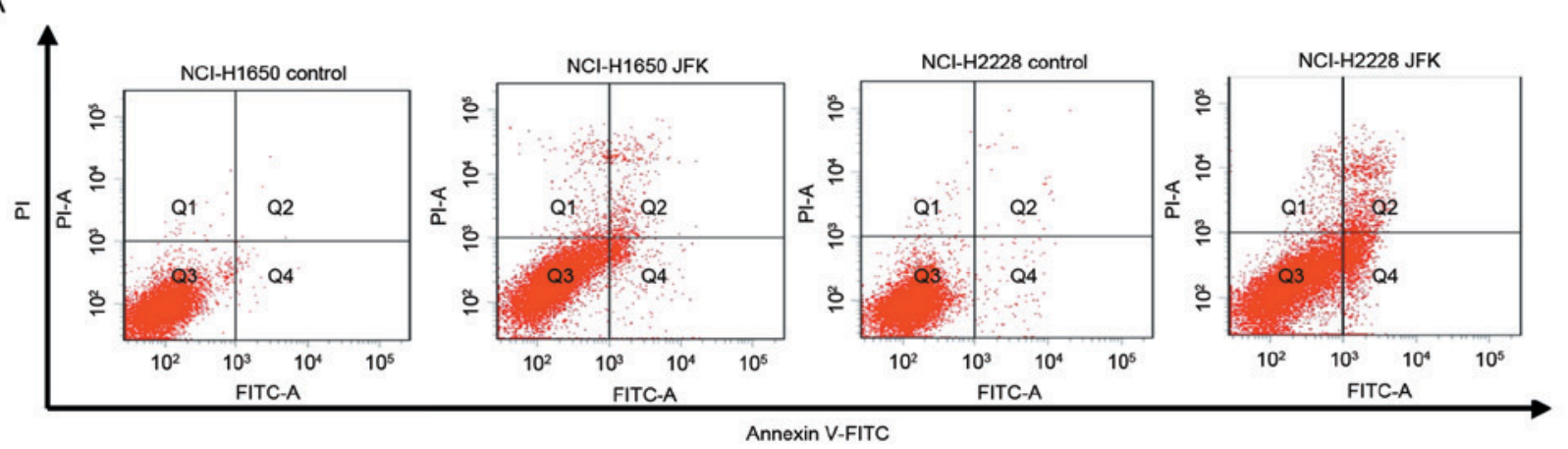

B

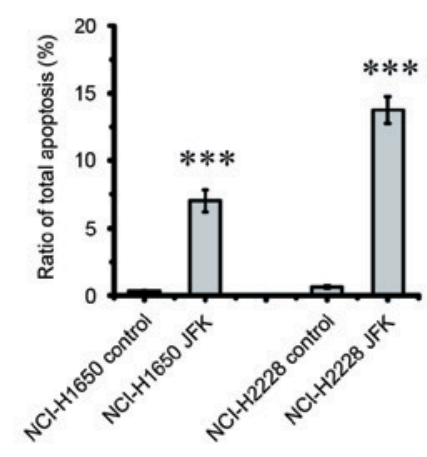

C

D
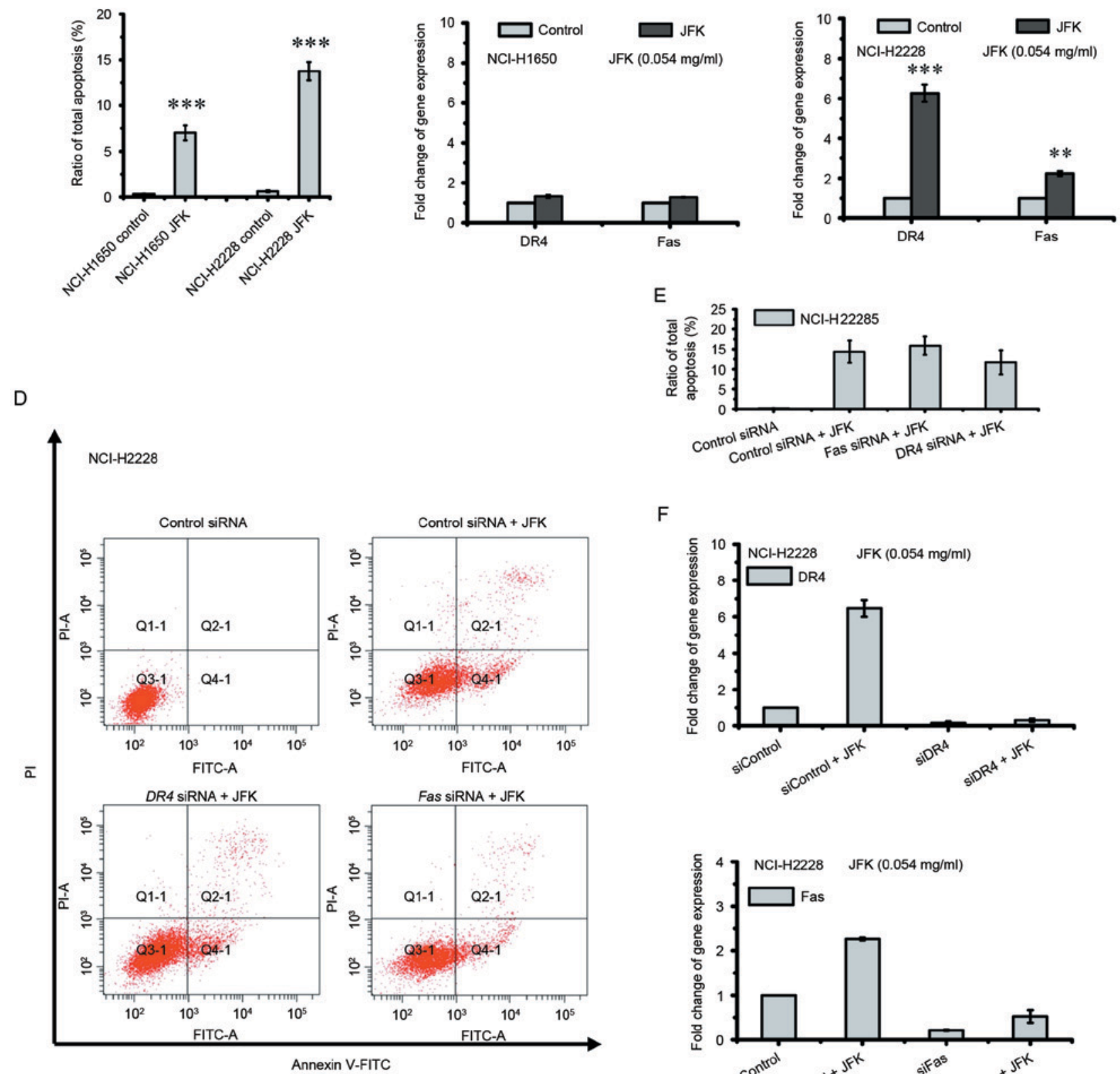

$\mathrm{F}$
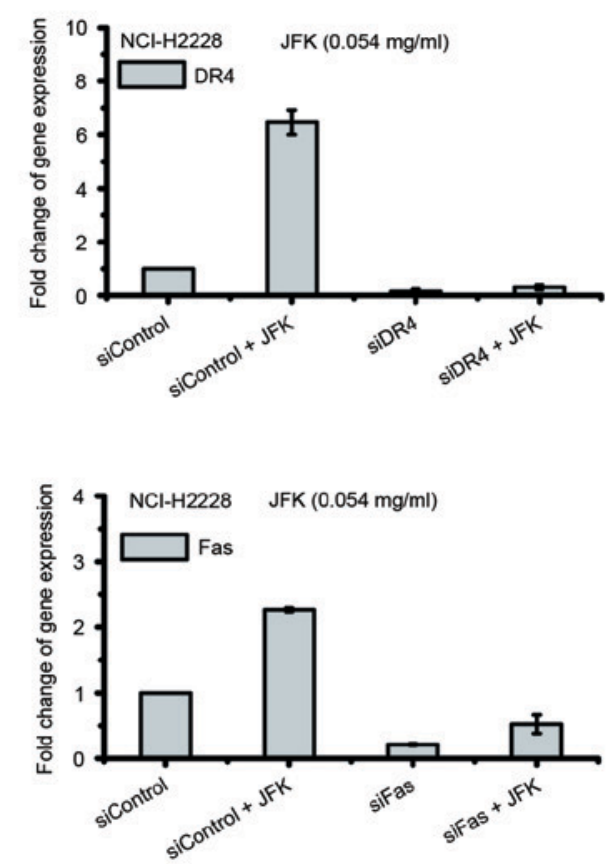

Figure 5. DR4 and Fas are not involved in JFK-induced apoptosis in NCI-H1650 and NCI-H2228 cells. (A) The apoptotic cells were detected by flow cytometry, after JFK $(0.054 \mathrm{mg} / \mathrm{ml})$ treatment for $48 \mathrm{~h}$ in NCI-H1650 and NCI-H2228 cells. (B) Apoptosis rate was analyzed based on flow cytometry. Data were expressed as the mean $\pm \mathrm{SD}(\mathrm{n}=3),{ }^{* * * *} \mathrm{P}<0.001$. (C) mRNA levels were examined by reverse transcription-quantitative polymerase chain reaction. Data were expressed as the mean $\pm \mathrm{SD}(\mathrm{n}=3),{ }^{* * *} \mathrm{P}<0.01$ and ${ }^{* * * *} \mathrm{P}<0.001$. (D and E) DR4 and Fas-knockdown NCI-H2228 cells were treated with JFK $(0.054 \mathrm{mg} / \mathrm{ml})$ for $48 \mathrm{~h}$, and then the percentage of apoptosis was determined by flow cytometry. Data are expressed as the mean \pm SD (n=3). (F) DR4 and Fas knockdown NCI-H2228 cells were treated with JFK $(0.054 \mathrm{mg} / \mathrm{ml})$ for $48 \mathrm{~h}$, and then the mRNA levels were examined by reverse transcription-quantitative polymerase chain reaction. Data are expressed as the mean $\pm \mathrm{SD}(\mathrm{n}=3)$. SD, standard deviation; DR4, death receptor 4; JFK, Jinfukang; siRNA, small interfering RNA; PI, propidium iodide; FITC, fluorescein isothiocyanate. 
transcriptome profiling analysis identified 5,281 genes whose expression was modulated by JFK and it was demonstrated that JFK contributes to A549 cellular apoptosis partially through activation of DR4 and Fas. The present results provide new insight for understanding how this TCM formula exerts its effects against lung cancer.

\section{Acknowledgements}

The authors would like to thank Professor Daniel M.Czajkowsky (School of Biomedical Engineering and Bio-ID Center, Shanghai Jiao Tong University, Shanghai, China) for helpful discussion and careful proofreading. The present study was supported by Longhua Medical Project of State Clinical Research Center of TCM in Longhua Hospital (grant nos. LYTD-21 and JDZX2012123), National Basic Research Program of China (grant no. 2013CB967402) and National Natural Science Foundation of China (grant nos. 81373623 and 91229123).

\section{References}

1. Li C and Hong W: Research status and funding trends of lung cancer biomarkers. J Thorac Dis 5: 698-705, 2013.

2. Siegel RL, Miller KD and Jemal A: Cancer statistics, 2015. CA Cancer J Clin 65: 5-29, 2015.

3. Ettinger DS, Wood DE, Akerley W, Bazhenova LA, Borghaei H, Camidge DR, Cheney RT, Chirieac LR, D'Amico TA, Demmy TL, et al: Non-small cell lung cancer, version 1.2015. J Natl Compr Canc Netw 12: 1738-1761, 2014.

4. Shanker M, Willcutts D, Roth JA and Ramesh R: Drug resistance in lung cancer. Lung Cancer (Auckl) 1: 23-36, 2010.

5. Efferth T, Li PC, Konkimalla VS and Kaina B: From traditional Chinese medicine to rational cancer therapy. Trends Mol Med 13: 353-361, 2007.

6. Lu J, Xu J and Shi Q: Effect of ethanol extract of HPRS, a Traditional Chinese Medicine formula, on HCT116 cell line. J Tradit Chin Med 36: 760-767, 2016.

7. Han R: Highlight on the studies of anticancer drugs derived from plants in China. Stem Cells 12: 53-63, 1994.

8. Jiao L, Wang Y, Xu L and You M: Lung cancer prevention and therapy using the JinFuKang herbal mixture. Curr Pharmacol Rep 1: 346-353, 2015.

9. Sun JL and Liu JX: Effect of 'Jinfukang Oral Solution' on expression of apoptosis related genes of human lung adenocarcinoma cells transplanted in nude mice. Shanghai J Tradit Chin Med 10, 2007.

10. Liu JX, Shi ZM, Li HG, Xu ZY, Zhu YW, Zhao LH, Gao H, Liu LS, Zhu HR and Zhang H: Clinical observation on 271 cases of non-small cell lung cancer treated with Yifei Kangliu Yin. Chin J Integr Tradit West Med 7: 247-250, 2001.

11. Xue R, Fang Z, Zhang M, Yi Z, Wen C and Shi T: TCMID: Traditional Chinese medicine integrative database for herb molecular mechanism analysis. Nucleic Acids Res 41 (Database issue): D1089-D1095, 2013.

12. Bonham M, Arnold H, Montgomery B and Nelson PS: Molecular effects of the herbal compound PC-SPES: Identification of activity pathways in prostate carcinoma. Cancer Res 62: 3920-3924, 2002.

13. Lu J, Chen J, Xu N, Wu J, Kang Y, Shen T, Kong H, Ma C, Cheng M, Shao Z, et al: Activation of AIFM2 enhances apoptosis of human lung cancer cells undergoing toxicological stress. Toxicol Lett 258: 227-236, 2016.

14. Pickrell JK, Marioni JC, Pai AA, Degner JF, Engelhardt BE, Nkadori E, Veyrieras JB, Stephens M, Gilad Y and Pritchard JK: Understanding mechanisms underlying human gene expression variation with RNA sequencing. Nature 464: 768-772, 2010.
15. Wang Z, Gerstein M and Snyder M: RNA-Seq: A revolutionary tool for transcriptomics. Nat Rev Genet 10: 57-63, 2009.

16. Lu J, Zhang X, Shen T, Ma C, Wu J, Kong H, Tian J, Shao Z, Zhao $X$ and $\mathrm{Xu}$ L: Epigenetic profiling of H3K4Me3 reveals herbal medicine Jinfukang-induced epigenetic alteration is involved in anti-lung cancer activity. Evid Based Complement Alternat Med 2016: 13, 2016.

17. Qi Y, Zhang X, Kang Y, Wu J, Chen J, Li H, Guo Y, Liu B, Shao $\mathrm{Z}$ and Zhao $\mathrm{X}$ : Genome-wide transcriptional profiling analysis reveals annexin A6 as a novel EZH2 target gene involving gastric cellular proliferation. Mol Biosyst 11: 1980-1986, 2015.

18. Trapnell C, Roberts A, Goff L, Pertea G, Kim D, Kelley DR, Pimentel H, Salzberg SL, Rinn JL and Pachter L: Differential gene and transcript expression analysis of RNA-seq experiments with TopHat and Cufflinks. Nat Protoc 7: 562-578, 2012.

19. Huang da W, Sherman BT and Lempicki RA: Systematic and integrative analysis of large gene lists using DAVID bioinformatics resources. Nat Protoc 4: 44-57, 2009.

20. Kanehisa M and Goto S: KEGG: Kyoto encyclopedia of genes and genomes. Nucleic Acids Res 28: 27-30, 2000.

21. Hesketh T and Zhu WX: Health in China. Traditional Chinese medicine: One country, two systems. BMJ 315: 115-117, 1997

22. Gordaliza M: Natural products as leads to anticancer drugs. Clin Transl Oncol 9: 767-776, 2007.

23. Cassileth BR, Rizvi N, Deng G, Yeung KS, Vickers A, Guillen S, Woo D, Coleton M and Kris MG: Safety and pharmacokinetic trial of docetaxel plus an Astragalus-based herbal formula for non-small cell lung cancer patients. Cancer Chemother Pharmacol 65: 67-71, 2009.

24. Nigg EA: Cyclin-dependent protein kinases: Key regulators of the eukaryotic cell cycle. Bioessays 17: 471-480, 1995.

25. Chohan TA, Qian H, Pan Y and Chen JZ: Cyclin-dependent kinase-2 as a target for cancer therapy: Progress in the development of CDK2 inhibitors as anti-cancer agents. Curr Med Chem 22: 237-263, 2015.

26. Vermeulen K, Van Bockstaele DR and Berneman ZN: The cell cycle: A review of regulation, deregulation and therapeutic targets in cancer. Cell Prolif 36: 131-149, 2003.

27. Volm M, Koomägi R, Mattern J and Stammler G: Cyclin $\mathrm{A}$ is associated with an unfavourable outcome in patients with non-small-cell lung carcinomas. Br J Cancer 75: 1774-1778, 1997.

28. Soria JC, Jang SJ, Khuri FR, Hassan K, Liu D, Hong WK and Mao L: Overexpression of cyclin B1 in early-stage non-small cell lung cancer and its clinical implication. Cancer Res 60: 4000-4004, 2000

29. Ouyang L, Shi Z, Zhao S, Wang FT, Zhou TT, Liu B and Bao JK: Programmed cell death pathways in cancer: A review of apoptosis, autophagy and programmed necrosis. Cell Prolif 45: 487-498, 2012.

30. Oh SB, Hwang CJ, Song SY, Jung YY, Yun HM, Sok CH, Sung HC, Yi JM, Park DH, Ham YW, et al: Anti-cancer effect of tectochrysin in NSCLC cells through overexpression of death receptor and inactivation of STAT3. Cancer Lett 353: 95-103, 2014.

31. Kobayashi S, Kajino S, Takahashi N, Kanazawa S, Imai K, Hibi Y, Ohara H, Itoh M and Okamoto T: 53BP2 induces apoptosis through the mitochondrial death pathway. Genes Cells 10: 253-260, 2005.

32. Fei P, Wang W, Kim Sh, Wang S, Burns TF, Sax JK, Buzzai M, Dicker DT, McKenna WG, Bernhard EJ and El-Deiry WS: Bnip3L is induced by p53 under hypoxia, and its knockdown promotes tumor growth. Cancer Cell 6: 597-609, 2004.

This work is licensed under a Creative Commons Attribution-NonCommercial-NoDerivatives 4.0 International (CC BY-NC-ND 4.0) License. 